\section{Mitochondrial ATP generation in stimulated platelets is essential for granule secretion but dis- pensable for aggregation and procoagulant activity}

Platelets, although anucleate, contain well-coupled and functional mitochondria ${ }^{1,2}$ and are capable of mitochondrial oxidative phosphorylation (OXPHOS). ${ }^{3}$ Simultaneous inhibition of glycolysis and OXPHOS abolishes agonistinduced aggregation and granule secretion. ${ }^{3}$ Recent studies have shown that there is considerable plasticity in energy metabolism of platelets. ${ }^{1}$ Yet, we demonstrated a switch to aerobic glycolysis from OXPHOS in stimulated platelets. ${ }^{2}$ Small-molecules that divert the flux from aerobic glycolysis towards OXPHOS prevent platelet activation. ${ }^{2}$ In addition, several studies including our own have established that inhibitors of OXPHOS alone do not compromise platelet aggregation. ${ }^{1,2,4}$ These observations led us to question whether mitochondrial ATP generation is dispensable for platelet function.

We undertook a comprehensive study of the effect of mitochondrial inhibitors (antimycin, oligomycin) and an uncoupler (CCCP; carbonyl cyanide 3-chlorophenylhydrazone) on platelet activity. We also established the probable mechanistic basis underlying their effects on platelet function. The underpinning modes of action for these molecules differ considerably. Antimycin acts by inhibiting complex III of the electron transport chain, thereby preventing both oxidation of energy substrates (Online Supplementary Figure S1A) as well as ATP synthesis. Oligomycin targets $\mathrm{F}_{0} \mathrm{~F}_{1}$ ATPase (complex V) leading to a block in ATP generation although oxidation of fuels continues to the extent allowed by proton leak (Online Supplementary Figure S1A). CCCP uncouples mitochondrial respiration and OXPHOS by dissipating the proton gradient (Online Supplementary Figure $S 1 B, C$ ) thus permitting maximal oxidation of fuels (Online Supplementary Figure $S 1 A$ ) albeit compromising formation of ATP. Hence, it is reasonable to expect that the effects of these molecules will not be identical. Since only oligomycin specifically and uniquely inhibits mitochondrial ATP generation, our inferences about the role of mitochondrial ATP in platelet function are based primarily on observations with oligomycin.

Platelet aggregation induced by either thrombin (Figure 1A, B) or collagen (Online Supplementary Figure S1D, G) was profoundly impaired in the presence of CCCP while the presence of antimycin or oligomycin did not appear to have a significant effect, which was consistent with earlier reports. ${ }^{1,2,4}$ Furthermore, we found similar results with aspirinated platelets (Online Supplementary Figure $S 1 E, I)$, suggesting that the effect of CCCP is likely independent of thromboxane A2 generation. Aggregation is mediated by fibrinogen which binds with high affinity to platelet surface integrin $\alpha_{\mathrm{IIb}} \beta_{3}$ in an active conformation. In keeping with our observation on platelet aggregation, CCCP brought about a significant drop in thrombininduced $\alpha_{\mathrm{II}} \beta_{3}$ integrin activation while antimycin and oligomycin had no such influence (Figure 1D, G).

A fraction of stimulated platelets, characterized by expression of phosphatidylserine on the outer leaflet of the plasma membrane, provide a procoagulant surface for generation of a fibrin clot at the site of vessel injury. ${ }^{5}$ The extent of phosphatidylserine exposure (Figure 1E, H) upon thrombin challenge was significantly retarded by CCCP but unaffected by either antimycin or oligomycin. These observations led us to deduce that mitochondrial ATP generation is dispensable for platelet integrin activation, aggregation and procoagulant activity. Nevertheless, disruption of mitochondrial membrane potential by CCCP impeded these processes.

Platelet responses to agonist stimulation are energydemanding. ${ }^{3,6}$ However, the energy requirements differ considerably, with shape change being the least energyrequiring process, followed by aggregation, dense/ $\alpha$ granule release and acid hydrolase secretion, in increasing order. ${ }^{3,7}$ We reasoned that inhibition of mitochondrial ATP generation could affect greater energy-intensive processes, such as exocytosis of granule contents. Release of adenine nucleotides and surface expression of P-selectin are markers of dense and $\alpha$ granule secretion, respectively. ATP release from platelets stimulated with either thrombin (Figure 1A, C) or collagen (Online Supplementary Figure $S 1 D, H)$, as well as P-selectin externalization induced by thrombin (Figure 1F, I) were significantly compromised in the presence of oligomycin and CCCP. Antimycin had no significant effect on either dense or $\alpha$ granule secretion (Figure 1A, C, F, I; Online Supplementary Figure $S 1 D, H)$, which was indicative of greater compensation by glycolytic ATP generated in the presence of antimycin (Figure $3 \mathrm{C}$ ). Glycolysis rate is enhanced in antimycin-treated platelets in order to compensate for significant inhibition in oxidation of fuels. ${ }^{4}$ Contrasting this, either residual oxidation allowed in mitochondria of oligomycin-treated platelets, or maximal oxidation provoked by CCCP, could restrict the compensatory increase in glycolysis and ensuing ATP availability, thus failing to sustain platelet functions with higher energy cost, such as granule secretion.

Expression of P-selectin on the surface of activated platelets triggers its interaction with the counter-ligand $\mathrm{P}$ selectin glycoprotein ligand-1 (PSGL-1) present on leukocytes. ${ }^{8}$ Hence, we measured platelet-leukocyte aggregates in whole blood challenged with thrombin receptor-activating peptide (TRAP)- 6 by flow cytometry. Both oligomycin and CCCP inhibited TRAP-stimulated platelet-neutrophil interaction (Online Supplementary Figure $S 2 A-E, K)$, which could be attributable to impaired $\mathrm{P}$-selectin externalization in these platelets. However, only CCCP was able to prevent TRAP-induced plateletmonocyte interaction (Online Supplementary Figure S2F-J, $L)$. Given that thrombin-induced phosphatidylserine exposure on platelets was unaffected by oligomycin, a phosphotidylserine-Tim 4 interaction ${ }^{9}$ could be mediating the platelet-monocyte interaction in oligomycin-treated platelets despite compromised P-selectin-facilitated interactions. This hypothesis was supported by our observation that oligomycin inhibited platelet-monocyte interactions (Online Supplementary Figure S1L) upon stimulation with ADP which, unlike TRAP, does not induce phosphatidylserine exposure.

Several prothrombotic factors are released from platelet granules upon stimulation, including $\mathrm{ADP}$ and fibrinogen..$^{10} \mathrm{ADP}$, in particular, plays a pivotal role in recruiting platelets to the 'shell' region of a growing thrombus. ${ }^{11}$ As we found that mitochondrial ATP sustained granule release, we hypothesized that it could be essential for the process of thrombosis. We studied platelet thrombus formation on immobilized collagen under arterial shear $\left(1500 \mathrm{~s}^{-1}\right)$. Washed human platelets were allowed to perfuse over the collagen-coated surface for $5 \mathrm{~min}$. Total surface area covered by platelet thrombi was significantly diminished in the presence of oligomycin (Figure 2A, E) and CCCP (Figure 2A, F) but unaffected by antimycin (Figure 2A, D), which could be attributable to impaired ADP release consequent to oligomycin and CCCP treatment. This hypothesis was supported by the observation that, neither oligomycin 
nor CCCP had any impact on thrombus growth in the presence of ticagrelor, an ADP receptor antagonist (Figure 2B, G-I). Furthermore, adhesion and spreading of platelets pretreated with tirofiban, an $\alpha_{\mathrm{II}} \beta_{3}$ integrin blocker, on collagen matrix under shear were unaffected in the presence of antimycin, oligomycin or CCCP (Figure
2C, J-L). Thus, ATP sourced from mitochondria was dispensable for platelet aggregation, adhesion and procoagulant activity but was essential for granule secretion, platelet-neutrophil interactions, as well as thrombus growth.

We asked whether differential modulation of platelet
A

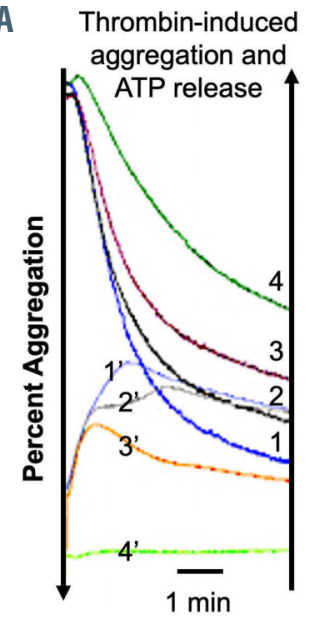

D

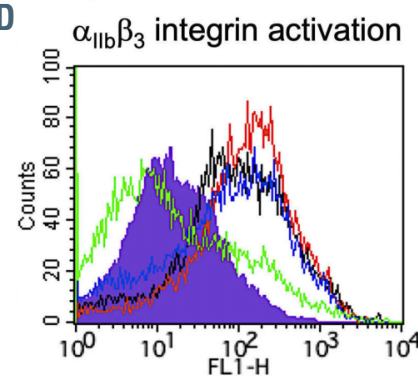

FITC-PAC1

RP

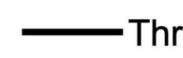

G

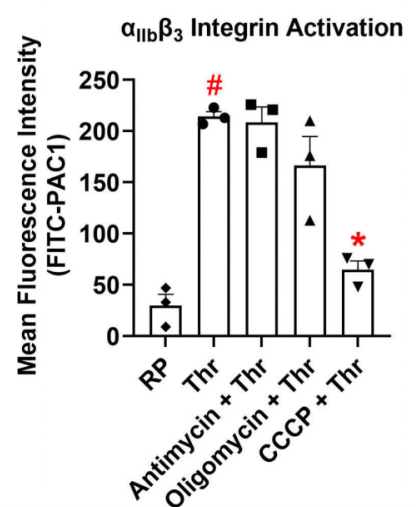

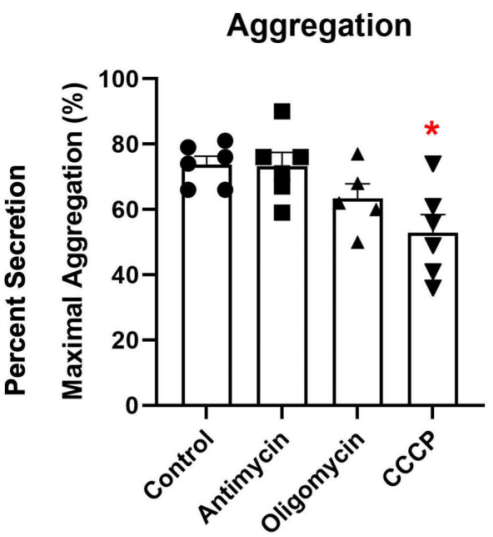

$E$

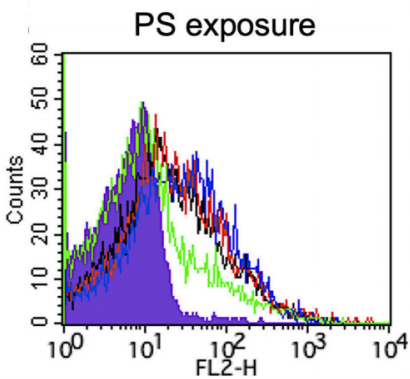

PE-Annexin V

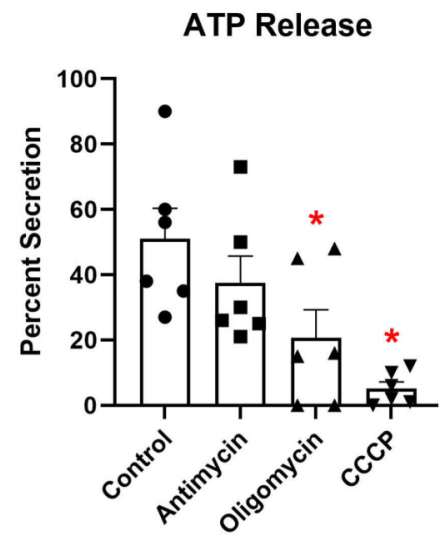

$\mathrm{F}$

P-selectin expression

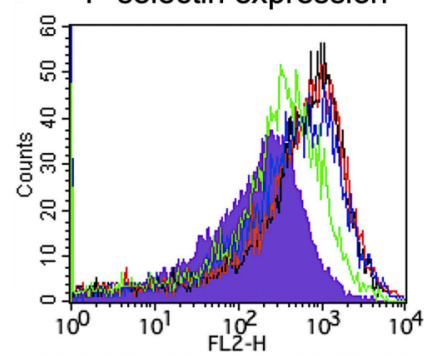

PE-Anti CD62P CCCP + Thr
$\mathrm{H}$

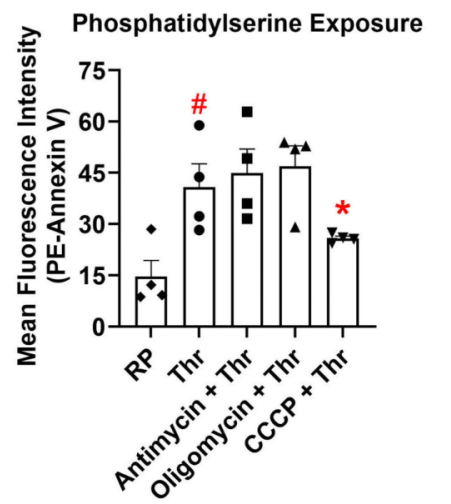

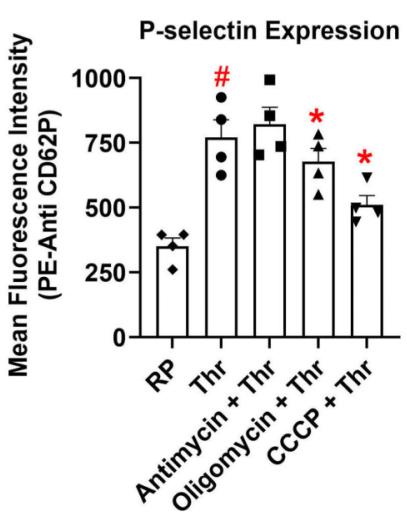

Figure 1. Effects of pre-treatment with mitochondrial inhibitors (antimycin, oligomycin) or an uncoupler (CCCP) on human platelet function in vitro. Washed human platelets were pre-treated with vehicle (control), antimycin $(2 \mu \mathrm{g} / \mathrm{mL})$, oligomycin $(10 \mu \mathrm{g} / \mathrm{mL})$ or CCCP $(100 \mu \mathrm{M})$ for $15 \mathrm{~min}$ at room temperature, followed by addition of human thrombin $(0.5 \mathrm{U} / \mathrm{mL})$ for $30 \mathrm{~min}$, or as indicated. (A) Representative tracings showing thrombin $(0.2 \mathrm{U} / \mathrm{mL})$-induced aggregation $(1-4)$ or ATP release (1'-4') of platelets pre-treated with vehicle (1,1'), antimycin (2,2'), oligomycin (3,3') or CCCP (4,4'). (B, C) Bar diagram quantifying mean platelet aggregation and platelet dense granule secretion in different samples, respectively. (D-F) Representative histogram overlay plots showing binding of FITC-PAC1 (BD Biosciences \#340507), PE-annexin V (Biolegend \#640908), and PE-anti-CD62P (BD Biosciences \#550561), respectively. (G-I) Corresponding bar diagrams quantifying $\alpha_{\| 1} \beta_{3}$ integrin activation, phosphatidylserine exposure and P-selectin expression, respectively. Each dot represents an independent observation. Data are presented as mean \pm standard error of mean. ${ }^{\star} P<0.05$ with respect to vehicle-treated thrombin-stimulated platelets. ${ }^{*} P<0.05$ with respect to vehicletreated unstimulated platelets. Significance in difference of means was tested by repeated measures analysis of variance and the Dunnett multiple comparison test. PS: phosphatidylserine; Thr: thrombin. 
bioenergetics by the uncoupler and inhibitors accounts for differences in their impact on platelet responses. Concurrent inhibition of both mitochondrial respiration and glycolysis has been found to impede platelet aggregation. ${ }^{1,2,4}$ This prompted us to hypothesize that, CCCP could be compromising both mitochondrial and glycolytic ATP generation, thereby leading to impaired platelet responses. Intriguingly, neither antimycin/oligomycin nor CCCP had any significant effect on cellular ATP level in platelets (Figure 3A). Sustained ATP levels suggest
A

Vehicle

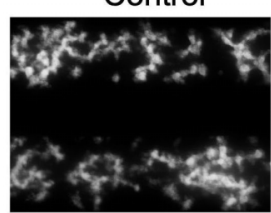

Antimycin

B

Ticagrelor

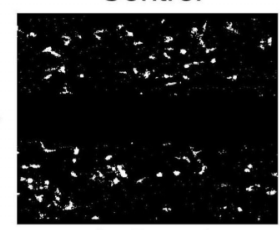

C

Tirofiban

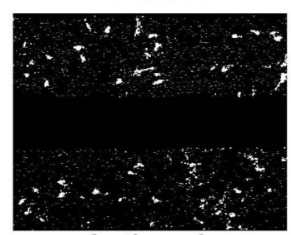

Antimycin
Control

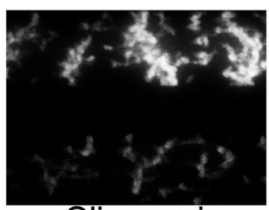

Oligomycin

Control

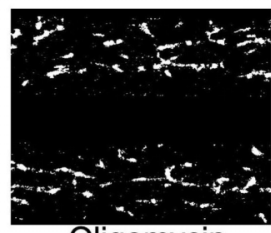

Oligomycin

Control

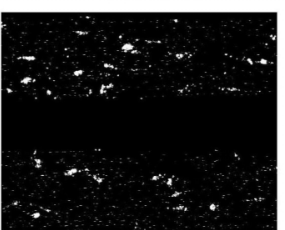

Oligomycin

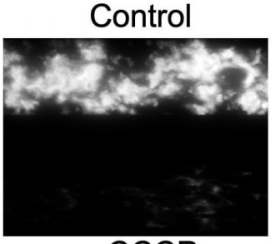

CCCP

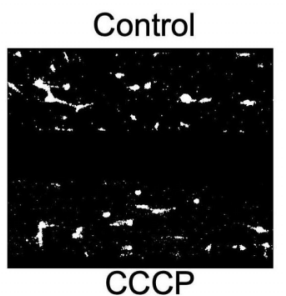

Control

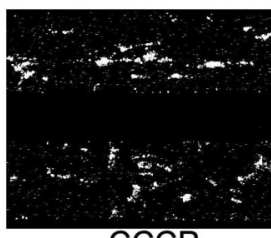

CCCP
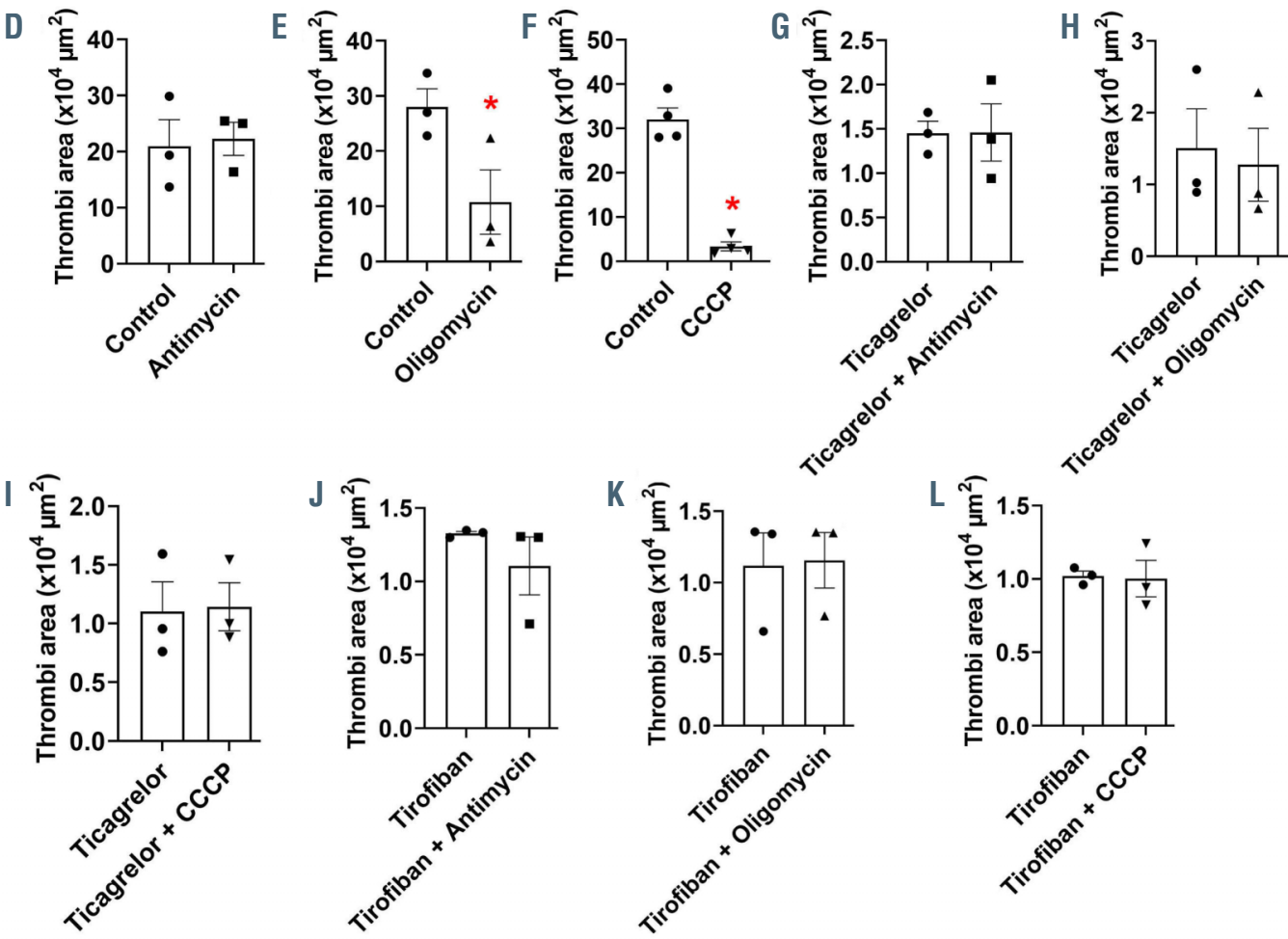

Figure 2. Effects of pre-treatment with mitochondrial inhibitors (antimycin, oligomycin) or an uncoupler (CCCP) on platelet thrombus formation. Washed human platelets pretreated with antimycin $(2 \mu \mathrm{g} / \mathrm{mL})$, oligomycin $(10 \mu \mathrm{g} / \mathrm{mL})$, CCCP $(100 \mu \mathrm{M})$ or vehicle (control) were labeled with calcein-AM (Molecular Probes \#C3100MP) and perfused over an immobilized collagen matrix for $5 \mathrm{~min}$ in a microfluidics flow chamber at a shear rate of $1500 \mathrm{~s}^{-1}$. (A-C) Representative images of platelet accumulation after $5 \mathrm{~min}$ of perfusion of human platelets with treatments as indicated in the presence of vehicle $(\mathrm{A})$, ticagrelor $(1 \mu \mathrm{M})(\mathrm{B})$ or tirofiban $(100 \mathrm{ng} / \mathrm{mL})(C)$. (D-L) Corresponding bar diagrams representing total surface area covered by platelet thrombi after 5 min of perfusion on the collagen matrix. Each dot represents an independent observation. Data are presented as mean \pm standard error of mean. $* P<0.05$ with respect to vehicle-treated sample. Statistical significance of difference of means was tested by a multiple paired Student $t$-test. 
compensatory augmentation in glycolysis. In agreement, treatment with either antimycin/oligomycin or CCCP was associated with enhanced lactate release from thrombin-stimulated platelets (Figure 3C), with antimycin being the most effective among them. These findings are strongly suggestive of a compensatory surge in glycolytic rate in platelets upon mitochondrial inhibition which sustains $\alpha_{\mathrm{II}} \beta_{3}$ platelet integrin activation, aggregation and phosphatidylserine exposure. Aggregation induced by threshold concentrations of thrombin and collagen was unaffected by oligomycin pre-treatment (Online Supplementary Figure S1F, $J, K$ ), which further bolsters the hypothesis that glycolytic ATP is necessary and sufficient to fuel platelet aggregation.

The above observations led us to consider that the molecular events underpinning the effects of CCCP on
A

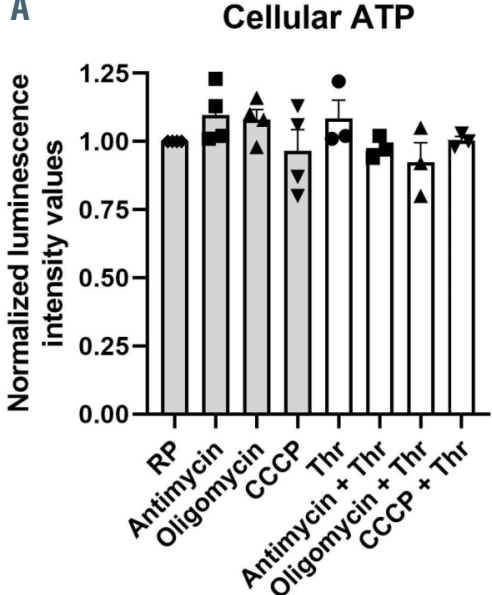

B

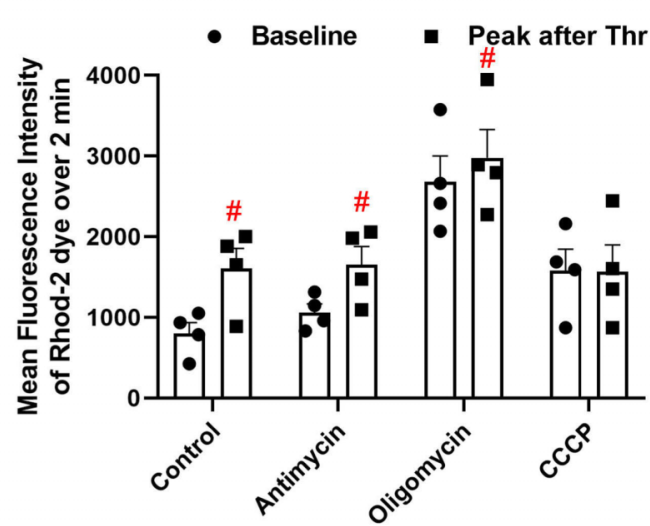

E

Mitochondrial Calcium $\circ$

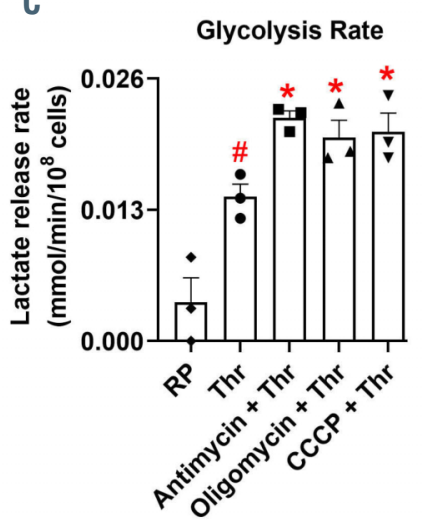

F

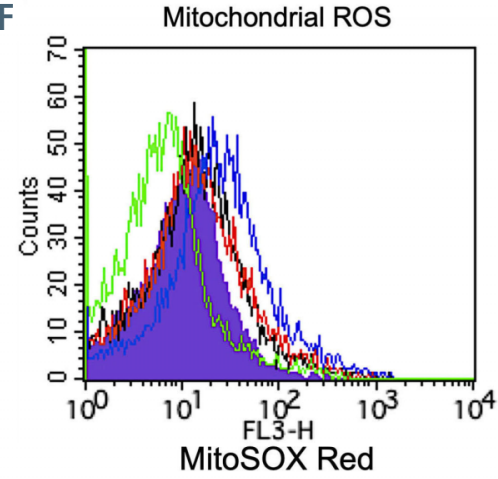

D

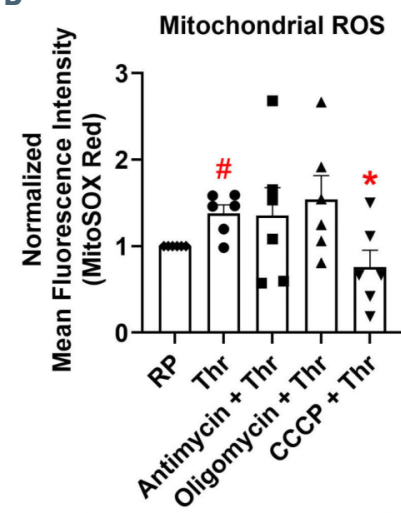

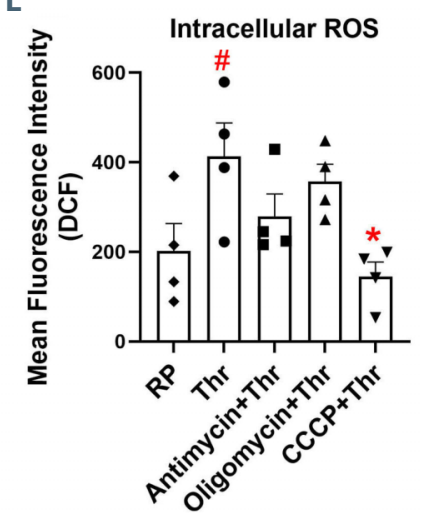

G

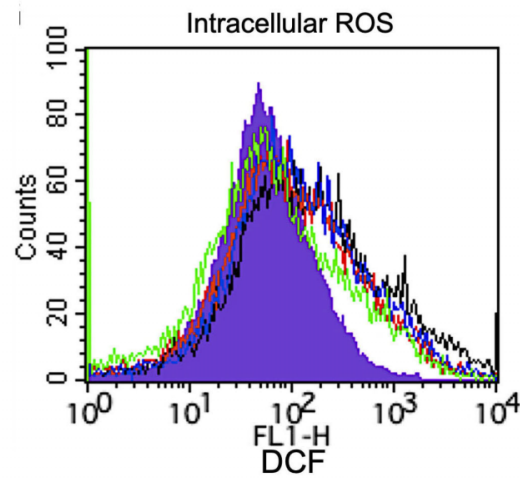

Oligomycin

$+\mathrm{Thr}$

$\mathrm{CCCP}$

Antimycin $+\mathrm{Thr}$

Figure 3. Effects of pre-treatment with mitochondrial inhibitors (antimycin, oligomycin) or an uncoupler (CCCP) on platelet ATP, reactive oxygen species, calcium and lactate release rates. Washed human platelets were pre-treated with vehicle (control), antimycin $(2 \mu \mathrm{g} / \mathrm{mL})$, oligomycin $(10 \mu \mathrm{g} / \mathrm{mL})$ or CCCP $(100 \mu \mathrm{M})$ for $15 \mathrm{~min}$ at room temperature, followed by addition of thrombin $(0.5 \mathrm{U} / \mathrm{mL}$ ) for $5 \mathrm{~min}$ (mitochondrial calcium), $15 \mathrm{~min}$ (cellular ATP), or $30 \mathrm{~min}$ (lactate release rates, mitochondrial reactive oxygen species [ROS] and intracellular ROS). (A-C) Bar diagrams indicating cellular ATP levels, mitochondrial calcium and lactate release rates, respectively. $(\mathrm{F}, \mathrm{G})$ Histogram overlay plots representing MitoSOX (Invitrogen \#M36008) and dichlorofluorescein (DCF) (Sigma \#D6883) fluorescence intensities, respectively. (D, E) Corresponding bar diagrams indicating mitochondrial and intracellular ROS levels, respectively. Each dot represents an independent observation. Data are presented as mean \pm standard error of mean ${ }^{*} P<0.05$ with respect to vehicle-treated thrombin-stimulated platelets. ${ }^{*} P<0.05$ with respect to vehicle-treated unstimulated platelets. Statistical significance of difference of means was tested by repeated measures analysis of variance and the Dunnett multiple comparison test. RP: resting platelets; Thr, thrombin. 
platelets could be mediated through mitochondrial functions unrelated to ATP generation. Reactive oxygen species (ROS) have now been established as important signaling molecules responsible for platelet activation. ${ }^{2,12}$ Mitochondria are a significant source of ROS in platelets ${ }^{12}$ and selectively scavenging mitochondrial superoxide with mitoTEMPO prevents platelet aggregation..$^{13}$ ROS release from mitochondria is dependent on inner mitochondrial membrane polarization, and uncoupling is known to reduce mitochondrial ROS. ${ }^{14}$ In concurrence, CCCP but not antimycin or oligomycin brought about a significant drop in mitochondrial ROS in thrombin-stimulated platelets (Figure 3D, F). These changes were also reflected in intracellular ROS levels, which were significantly curbed in the presence of CCCP but remained unaffected by antimycin or oligomycin (Figure 3E, G). Phosphatidylserine exposure by platelets is dependent on cyclophilin D-dependent formation of mitochondrial permeability transition pores, which is triggered by calcium entry into mitochondria through a mitochondrial calcium uniporter along the electrical gradient across the inner mitochondrial membrane. ${ }^{15}$ We could find inhibition of thrombin-induced mitochondrial calcium transients in the presence of CCCP but not in the presence of antimycin or oligomycin (Figure 3B; Online Supplementary Figure $S 3 A-D)$. Hence, it was fairly reasonable to posit that CCCP restrains platelet aggregation and procoagulant activity through abrogation of mitochondrial ROS and calcium transients, respectively.

In summary, mitochondrial ATP was found to be dispensable for platelet aggregation and procoagulant activity, which are fueled by glycolytic ATP. However, maintenance of a proton gradient across inner mitochondrial membrane plays a vital role in these processes by supporting ROS generation and mitochondrial calcium influx, respectively. We discovered that mitochondrial ATP is critical for sustaining platelet granule secretion, platelet-neutrophil interactions and thrombus growth, especially when inadequately compensated by glycolytic ATP (Online Supplementary Figure S3E). This knowledge should have important implications for the development of anti-thrombotic strategies that selectively target platelet granule release in the treatment of thromboinflammatory diseases such as acute myocardial infarction, ischemic stroke, deep vein thrombosis and pulmonary embolism.

Paresh P. Kulkarni, ${ }^{1}$ Mohammad Ekhlak, ${ }^{1}$

Vijay K. Sonkar and Debabrata Dash'

${ }^{1}$ Center for Advanced Research on Platelet Signaling and Thrombosis Biology, Department of Biochemistry, Institute of Medical Sciences, Banaras Hindu University, Varanasi, Uttar Pradesh and ${ }^{2}$ Department of Molecular and Human Genetics, Institute of Science, Banaras Hindu University, Varanasi, Uttar Pradesh, India

Correspondence:

DEBABRATA DASH: ddash.biochem@gmail.com

doi:10.3324/haematol.2021.279847

Received: August 19, 2021.
Accepted: December 16, 2021.

Pre-published: December 23, 2021.

Disclosures: this research was supported by a J.C. Bose National Fellowship and grants received by DD from the Indian Council of Medical Research (ICMR) under CAR, Department of Biotechnology (DBT) and Science and Engineering Research Board (SERB), Government of India. DD also acknowledges assistance from the Humboldt Foundation, Germany. ME is a recipient of a CSIR-JRF

Contributions: DD supervised the entire work; DD and PPK designed the research; $P P K, M E$ and VKS performed experiments and analyzed results; DD and PPK wrote the manuscript.

\section{References}

1. Ravi S, Chacko B, Sawada H, et al. Metabolic plasticity in resting and thrombin activated platelets. PLoS One. 2015;10(4):e0123597.

2. Kulkarni PP, Tiwari A, Singh N, et al. Aerobic glycolysis fuels platelet activation: small-molecule modulators of platelet metabolism as anti-thrombotic agents. Haematologica. 2019;104(4):806818.

3. Holmsen H. Energy metabolism and platelet responses. Vox Sang. 1981;40(1):1-7.

4. Kaczara P, Sitek B, Przyborowski K, et al. Antiplatelet effect of carbon monoxide is mediated by NAD+and ATP depletion. Arterioscler Thromb Vasc Biol. 2020;40:2376-2390.

5. Nechipurenko DY, Receveur N, Yakimenko AO, et al. Clot contraction drives the translocation of procoagulant platelets to thrombus surface. Arterioscler Thromb Vasc Biol. 2019;39(1):3747.

6. Verhoeven AJM, Mommersteeg ME, Willem J, Akkerman N. Quantification of energy consumption in platelets during thrombin-induced aggregation and secretion. Tight coupling between platelet responses and the increment in energy consumption. Biochem J. 1984;221(3):777-787.

7. Holmsen H, Kaplan KL, Dangelmaier CA. Differential energy requirements for platelet responses. A simultaneous study of aggregation, three secretory processes, arachidonate liberation, phosphatidylinositol breakdown and phosphatidate production. Biochem J. 1982;208(1):9-18.

8. Kral JB, Schrottmaier WC, Salzmann M, Assinger A. Platelet interaction with innate immune cells. Transfus Med Hemother. 2016;43(2):78-88.

9. Segawa K, Nagata S. An apoptotic 'eat me' signal: phosphatidylserine exposure. Trends Cell Biol. 2015;25(11):639-650.

10. Golebiewska EM, Poole AW. Platelet secretion: from haemostasis to wound healing and beyond. Blood Rev. 2015;29(3):153-162.

11. Welsh JD, Stalker TJ, Voronov R, et al. A systems approach to hemostasis: 1 . The interdependence of thrombus architecture and agonist movements in the gaps between platelets. Blood. 2014;124(11):1808-1815

12. Masselli E, Pozzi G, Vaccarezza M, et al. ROS in platelet biology: functional aspects and methodological insights. Inty J Mol Sci. 2020;21(14):1-35.

13. Sonkar VK, Kumar R, Jensen M, et al. Nox2 NADPH oxidase is dispensable for platelet activation or arterial thrombosis in mice. Blood Adv. 2019;3(8):1272-1284

14. Cadenas S. Mitochondrial uncoupling, ROS generation and cardioprotection. Biochim Biophys Acta Bioenerg. 2018;1859(9):940950.

15. Kholmukhamedov A, Janecke R, Choo HJ, Jobe SM. The mitochondrial calcium uniporter regulates procoagulant platelet formation. J Thromb Haemost. 2018;16(11):2315-2321. 